\title{
Fatores Associados à Escolha da Especialidade de Medicina de Família e Comunidade
}

\author{
Factors Associated with the Choice of Specializing in Family Medicine
}

\section{PALAVRAS-CHAVE}

- Medicina de Família e Comunidade.

- Especialidade Médica.

- Educação Médica.

\author{
Lucia Helena Guimarães Rodrigues ${ }^{I}$ (iD \\ Taciana Barbosa Duque ${ }^{I}$ (D) \\ Reneide Muniz da Silva ${ }^{I}$ (iD
}

\section{RESUMO}

Introdução: A Medicina de Família e Comunidade (MFC) é considerada a especialidade mais relacionada com os princípios de longitudinalidade, integralidade e coordenação do cuidado da atenção primária à saúde (APS). A escolha da especialidade de MFC pode ser considerada por aspectos pessoais, profissionais e do currículo médico. O objetivo deste estudo foi analisar o perfil dos médicos residentes e egressos dos programas de residência de MFC de Pernambuco e os fatores associados à escolha da especialidade. Método: Realizou-se um estudo de corte transversal envolvendo 129 médicos ingressantes nas residências de MFC de Pernambuco, de 2012 a 2017. A coleta de dados foi feita com a aplicação de questionário eletrônico do LimeSurvey em escala tipo Likert de cinco pontos, com perguntas sobre fatores que contribuíram para a escolha da especialidade. Fizeram-se análises descritivas das variáveis do estudo no Epi Info 7.0, apresentadas em distribuição de frequência e medidas de tendência central e dispersão. Avaliou-se a consistência interna dos dados pelo alfa de Cronbach. O estudo foi aprovado pelo Comitê de Ética em Pesquisa da Faculdade Pernambucana de Saúde. Resultados: Responderam ao questionário 104 médicos, com média de idade de $31 \pm 6,1$ anos. Do total de participantes, $58,6 \%$ eram mulheres, $70 \%$ eram egressos de faculdades públicas e $66,3 \%$ escolheram a MFC após a conclusão da faculdade. Os aspectos pessoais analisados como influenciadores na escolha da especialidade de MFC foram: compromisso social, aptidão e afinidade com a especialidade, e circunstâncias da vida pessoal. Em relação às características da residência, a duração e a disponibilidade de vagas favoreceram a escolha. A maior parte dos aspectos relacionados com as caraterísticas próprias da especialidade exerceu influência positiva na escolha dos participantes. Os fatores do currículo da graduação que favoreceram a escolha da especialidade foram: influência de preceptores modelos e atividades práticas no contexto da MFC. O coeficiente alfa de Cronbach foi de 0,847. Conclusões: Entre os fatores referidos pelos residentes como influenciadores na escolha da especialidade de MFC, incluem-se os aspectos pessoais e as características do programa de residência e da especialidade. Por sua vez, os aspectos do currículo da graduação, de forma geral, não contribuíram para a essa escolha, entretanto houve concordância de que a vivência em cenário de prática de MFC e o exemplo de preceptores modelos influenciaram na decisão. 


\section{KEYWORDS}

- Family Medicine.

- Medical Specialty.

- Medical Education.

Recebido em 14/4/20

Aceito em 21/4/20

\section{INTRODUÇÃO}

As Diretrizes Curriculares Nacionais (DCN) do curso de graduação em Medicina, seguindo a proposta de redefinição da formação médica da Organização Pan-Americana da Saúde (Opas), destacam que os cursos médicos precisam ampliar o enfoque na comunidade e inserir os estudantes desde o início da graduação e ao longo do curso nas redes de serviços de saúde, que se constituem em importantes ambientes de aprendizagem. As DCN apontam para uma formação médica mais geral, humanista e crítica com capacidade para atuar nos diferentes níveis de atenção à saúde, dando centralidade para o ensino da atenção básica organizado e coordenado pela área de Medicina de Família e Comunidade (MFC). A inserção do estudante em cenários de atenção primária deve ser contínua durante todo o curso médico. No período do internato, ao menos $30 \%$ da carga horária deve ser desenvolvida na atenção primária e em serviço de urgência e emergência do Sistema Único de Saúde (SUS), com predomínio da carga horária dedicada à atenção primária e voltada para a área de $\mathrm{MFC}^{1,2}$. Tal imersão social do estudante visa favorecer a construção das competências necessárias para que o cuidado integral dos pacientes se dê de forma interligada, permanente e contextualizada, sempre permeado pelo compromisso social ${ }^{3-6}$.

Para além da graduação, outra preocupação dos gestores públicos e da sociedade em geral recai sobre a futura especialidade a ser exercida pelos egressos dos cursos médicos brasileiros, uma vez que a má distribuição e a desproporção das especialidades impactam diretamente o funcionamento do sistema de saúde. Idealmente, a escolha da especialidade deveria resultar do equilíbrio entre o interesse do profissional e as necessidades dos cidadãos, uma vez que ela determina

\section{ABSTRACT}

Introduction: Family Medicine (FM) is considered the specialty which is most closely related to the primary health care (PHC) principles of longitudinality, comprehensiveness and coordination. The choice to specialize in FM can be related to personal, professional and curricular aspects. Objective: To analyze the profile of family medicine residents in Pernambuco and the factors associated with the choice of this specialty. Method: Cross-sectional study, involving 129 physicians entering FM residency in Pernambuco from 2012 to 2017. Data collection was performed by applying an electronic LimeSurvey questionnaire using a five-point Likert scale with questions about factors that may have contributed to their choice of the specialty. Descriptive analyses of the study variables were performed in EpiInfo ${ }^{\circ}$ 7.0, presented in frequency distribution and measures of central tendency and dispersion. The internal consistency of the data was assessed using Alpha Cronbach. The study was approved by the Research Ethics Committee of the Faculdade Pernambucana de Saude. Results: 104 physicians answered the questionnaire, with a mean age of $31 \pm 6.1$ years, 58.6\% women, $70 \%$ were graduate analysed in relation to influence on the physician's choice to specialize in FM are social commitment, aptitude, affinity with the specialty and personal life circumstances. In terms of the characteristics of medical residency, both the duration and the availability of places in the state's FM programs played a part in the choice. Most aspects related to the choice of following a career in FM were classed as a positive influence on that choice. Factors related to the undergraduate curriculum included model preceptors and practical activities in the family medicine setting. Cronbach's alpha coefficient was 0.847. Conclusion: The factors cited by residents as influencing their choice to specialize in FM include personal aspects, characteristics of the residency program as well as characteristics of the specialty itself. On the other hand, aspects related to the undergraduate curriculum in general did not contribute to this choice. There was, however, agreement that practical experience in a FM setting and the example of model tutors influenced the decision. 
tem sido encarada como um alerta para o governo e as escolas médicas ${ }^{19}$.

No âmbito da formação médica, observa-se que, a despeito do que é preconizado pelas DCN do curso de graduação em Medicina, a inserção dos graduandos na APS e MFC se dá de maneira heterogênea entre os cursos. Alguns estudos apontam que a especialidade de MFC tem sido mais valorizada, tanto por docentes como por discentes, nas instituições de ensino superior (IES), em que médicos de família compõem o corpo docente e os estudantes são precoce e frequentemente expostos à prática em APS, sobretudo quando os preceptores são modelos de médicos de família. Nessas condições, os estudantes podem se identificar mais facilmente com a especialidade durante o curso e ter maior chance de escolher a carreira de $\mathrm{MFC}^{20-27}$.

Diante da importância da MFC no contexto da APS e do SUS, este estudo se propõe a conhecer os fatores que influenciam os médicos na escolha da especialidade de MFC, sobretudo aqueles relacionados com o currículo da graduação.

\section{MÉTODO}

Realizou-se um estudo de corte transversal no estado de Pernambuco, no período de junho de 2017 a maio de 2018. A população do estudo foi composta de 129 médicos egressos e residentes dos programas de residência de MFC da Secretaria Estadual de Saúde de Pernambuco (SES-PE) que ingressaram entre 2012 e 2017. Até a conclusão do estudo, existiam quatro faculdades de Medicina no estado de Pernambuco com turmas já concluídas: três públicas - Universidade Federal de Pernambuco (Ufpe), Universidade Estadual de Pernambuco (UPE) e Universidade Federal do Vale do São Francisco (Univasf) - e uma privada - Faculdade Pernambucana de Saúde (FPS). Como a FPS teve sua primeira turma de Medicina concluída em 2011, optou-se por subdividir a amostra da pesquisa em dois grupos - médicos formados antes e a partir de 2011 -, a fim de comparar os resultados das quatro faculdades de forma igualitária. A coleta de dados foi realizada por meio da aplicação de um questionário eletrônico estruturado, composto por duas seções: 1 . perguntas sobre as características sociodemográficas e acadêmicas dos participantes e 2., em escala tipo Likert de cinco pontos, questões sobre os fatores que influenciaram na escolha da especialidade. Para cada assertiva da segunda seção, o candidato poderia escolher um dos cinco itens da escala: 1 - discordo fortemente; 2 - discordo; 3 - indiferente; 4 - concordo; 5 concordo fortemente. O questionário foi elaborado em formato eletrônico e enviado para o WhatsApp e/ou e-mail pessoal dos participantes por meio do programa LimeSurvey versão 2.0.

Os dados coletados foram armazenados no próprio LimeSurvey 2.0 e posteriormente organizados em planilha Excel. O programa utilizado para a análise estatística foi o Epi Info versão 7.0. Para analisar os fatores influenciadores da escolha da especialidade da escala de Likert, utilizou-se o ranking médio da pontuação, relacionado à frequência das respostas dos participantes que fizeram tal atribuição. Como a escala foi de cinco pontos, os valores maiores que três foram considerados concordantes. Calculouse o alfa de Cronbach para avaliar a consistência das variáveis analisadas. Fizeram-se análises descritivas das variáveis do estudo, apresentadas em distribuição de frequência, e medidas de tendência central e dispersão

A pesquisa foi aprovada pelo Comitê de Ética em Pesquisa da Faculdade Pernambucana de Saúde: Certificado de Apresentação para Apreciação Ética (CAEE) no 65565517.9.0000.5569.

\section{RESULTADO}

Do total de 129 médicos que ingressaram nos programas de residência de MFC do estado de Pernambuco, três foram excluídos do estudo por terem desistido da residência ao longo dos dois anos do programa. $\mathrm{O}$ índice de resposta obtido foi de 82,5\%, com 104 questionários respondidos de forma completa. A média de idade foi de $31 \pm 6,1$ anos, com três participantes abaixo dos 25 anos e dois acima dos 50 . Houve predomínio de mulheres (58,6\%). Em relação à cor, 51 (49\%) se declararam brancos e 51 (49\%) mencionaram que eram pardos ou pretos. Mais da metade dos participantes eram solteiros (51\%) e cerca de $80 \%$ não tinham filhos. Quanto à naturalidade, 58 (55,8\%) nasceram em cidades do interior do Brasil (Tabela 1).

\section{Tabela 1}

Características sociodemográficas de médicos que ingressaram no

programa de residência de Medicina de Família e Comunidade no estado de Pernambuco, no período de 2012 a 2017 - Recife, 2018

Características sociodemográficas $\mathrm{N}(104)$ $\%$

$<25$ anos

3

25-30 anos 54

31-40 anos 45

51,9

$>=51$ anos

2

43,3

exo

Feminino

61

58,6

Masculino

43

41,4

Estado civil

Solteiro 53

Casado/união estável 50

Separado/divorciado 1

Quantidade de filhos

0

1

2

3

4 ou mais 82

13

Raça/cor

Branca

51

Parda

Preta

Amarela

Indígena

1

Local de nascimento

Interior

58

Capital

46

Estado de nascimento

Pernambuco

60

Outros

44

42,3

Fonte: Elaborada pelas autoras 
Em relação à graduação, a maioria dos participantes cursou Medicina em faculdades públicas (72,1\%), e, dos 29 (27,9\%) que estudaram em faculdades privadas, $44,8 \%$ utilizaram o Fundo de Financiamento Estudantil (Fies) como forma de financiamento parcial ou total. Quanto ao momento da escolha da especialidade, 69 (66,3\%) médicos escolheram fazer a residência de MFC após a conclusão da faculdade (Tabela 2).

No período do estudo, 65 (62,5\%) eram residentes e 39 participantes (37,5\%) já haviam concluído a residência. Dos respondentes, $75 \%$

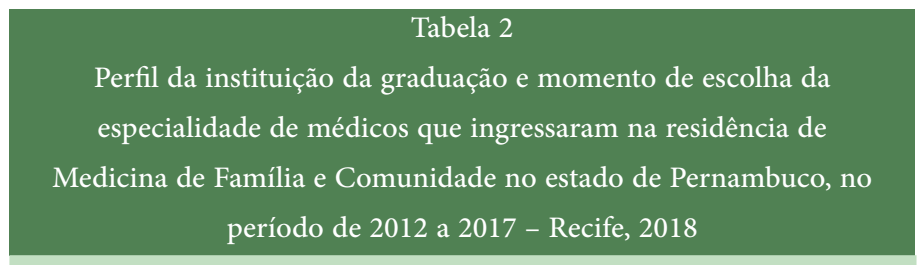

\begin{tabular}{|c|c|c|}
\hline Natureza jurídica da faculdade onde cursou Medicina & $\mathrm{n}$ & $\%$ \\
\hline Faculdade pública & 75 & 72,1 \\
\hline Faculdade privada & 29 & 27,9 \\
\hline TOTAL & 104 & 100 \\
\hline
\end{tabular}

Financiamento da faculdade privada

Recursos de membros da família

n $\%$

Fies

1241,3

Recursos de membros da família + Fies

Recursos próprios

$10 \quad 34,4$

Recursos próprios + membro da família

Programa Universidade par Todos (Prouni)

Outros

TOTAL

$03 \quad 10,3$

$01 \quad 3,5$

$01 \quad 3,5$

$01 \quad 3,5$

$01 \quad 3,5$

$29 \quad 100$

Ano de conclusão do curso

Antes de 2011

n $\quad \%$

A partir de 2011

$20 \quad 19,2$

TOTAL

Faculdade onde cursou medicina

(Concluintes a partir de 2011)

Faculdades de outros estados ou países

$84 \quad 80,8$

$104 \quad 100$

Univasf

UPE

FPS

n $\%$

Ufpe

TOTAL

Momento da escolha da especialidade

Antes de entrar na faculdade

Nos primeiros quatro anos da faculdade

2630,9

Durante o internato

Depois de concluir a faculdade

TOTAL

$\begin{array}{cc}26 & 30,9 \\ 20 & 23,8 \\ 13 & 15,5 \\ 13 & 15,5 \\ 12 & 14,3 \\ 84 & 100 \\ \mathrm{n} & \% \\ 02 & 1,9 \\ 11 & 10,6 \\ 22 & 21,2 \\ 69 & 66,3 \\ 104 & 100\end{array}$

Fonte: Elaborada pelas autoras. ingressaram na residência entre 2015 e 2017. Pouco mais de $10 \%$ dos participantes fizeram outra residência além de MFC (Tabela 3).

$\mathrm{Na}$ opinião dos participantes, os aspectos pessoais que influenciaram na escolha da especialidade foram: compromisso social, aptidão e afinidade com a especialidade, e circunstâncias da vida familiar ou pessoal. A opinião e o exemplo dos pais ou de outros parentes não foram relevantes na escolha da MFC.

Em relação às características da residência, tanto a duração quanto a disponibilidade de vagas nos programas de MFC do estado favoreceram a escolha da especialidade.

Quanto às características intrínsecas da própria especialidade, a maioria delas exerceu influência positiva na escolha dos participantes,

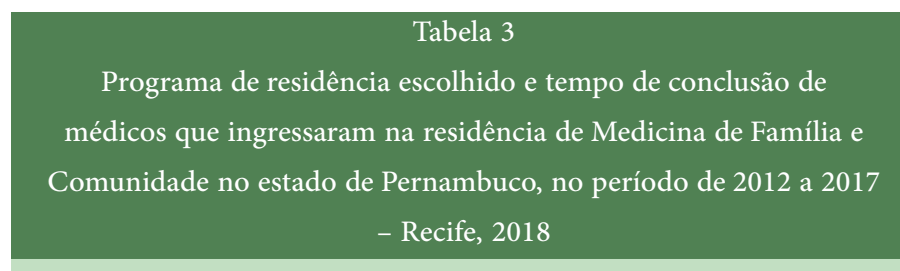

\begin{tabular}{|ccc|} 
Ano de ingresso na residência & $\mathrm{n}$ & $\%$ \\
2012 & 12 & 11,6 \\
2013 & 07 & 6,7 \\
2014 & 07 & 6,7 \\
2015 & 20 & 19,2 \\
2016 & 22 & 21,2 \\
2017 & 36 & 34,6 \\
TOTAL & 104 & 100 \\
\hline Instituição da residência de Medicina de Família e & $\mathrm{n}$ & $\%$
\end{tabular}
Comunidade

$\begin{array}{lcc}\text { Universidade do Vale do São Francisco (Univasf) } & 38 & 36,5 \\ \text { Prefeitura do Recife (PCR) } & 17 & 16,4 \\ \text { Universidade Estadual de Pernambuco (UPE) } & 14 & 13,5 \\ \text { Instituto de Medicina Integral Prof. Fernando } & 12 & 11,6 \\ \text { Figueira (Imip) } & & \end{array}$

Hospital das Clínicas da Ufpe (HC-Ufpe) $\quad 09$

Núcleo de Ciências da Vida/Ufpe - Caruaru $09 \quad 8,6$ (NCV-Ufpe)

$\begin{array}{lll}\text { Prefeitura de Jaboatão } & 05 & 4,8\end{array}$

\section{TOTAL}

Conclusão da residência

$104 \quad 100$

Sim

Não (residência em curso)

n $\%$

TOTAL

$39 \quad 37,5$

Outra residência além de MFC

$65 \quad 62,5$

Sim

Não

$12 \quad 11,5$

TOTAL

$104 \quad 100$ 
tais como: conteúdo técnico, perspectivas de crescimento profissional, oportunidade de ensino e pesquisa, diversidade de casos, atmosfera no ambiente de trabalho, oportunidade de realizar procedimentos ambulatoriais, carga horária média semanal, tipo de relação médicopaciente, trabalho em equipe multidisciplinar, estilo de vida que a especialidade propicia, assistência predominantemente ambulatorial, cuidado integral do paciente, longitudinalidade do cuidado, preferência por cuidado primário versus cuidado hospitalar, trabalho na comunidade $\mathrm{e}$ autonomia que a especialidade propicia. Em contrapartida, as características da especialidade que se destacaram como não influenciadoras foram: o prestígio social da MFC, o retorno financeiro, o mercado de trabalho, na perspectiva da segurança profissional, e a flexibilidade de horário.

No âmbito do currículo da graduação médica, apenas dois fatores foram apontados como tendo favorecido a escolha da especialidade: a influência de algum docente ou preceptor modelo e as atividades práticas vivenciadas no contexto da MFC durante os estágios. Os demais fatores do currículo, como disciplinas ou módulos relacionados com a MFC, atividades extracurriculares, participação em ligas acadêmicas, movimento estudantil e projetos de extensão ou de pesquisa, não exerceram papel positivo na escolha dos participantes (Tabela 4).

A aplicação do coeficiente alfa de Cronbach com valor de 0,847 demonstrou uma boa consistência das variáveis analisadas.

\section{DISCUSSÃO}

As crescentes mudanças nas demandas de saúde apontam a atenção primária e a MFC como estratégias de saúde eficazes em diversos países, entretanto ainda está longe de ser uma das principais escolhas entre os egressos dos cursos médicos no Brasil ${ }^{16,17}$. Estudos que abordam essa temática ainda são escassos. Com essa preocupação, estudamos a opinião de residentes e ex-residentes de MFC sobre os fatores que contribuíram para a escolha da especialidade.

Fatores que influenciaram na escolha da especialidade

O compromisso social foi decisivo para a escolha da especialidade de MFC na opinião dos médicos participantes do estudo. Mesmo sendo considerada uma característica pessoal que atuaria como motivação intrínseca na escolha profissional, entendemos que o compromisso social pode ser estimulado ou despertado ao longo do percurso da graduação médica. Na verdade, até mesmo anteriormente a isso, por meio da utilização de critérios de admissão nas escolas médicas que favoreçam estudantes com características socioeconômicas, culturais e geográficas diversas, pode-se aumentar o compromisso social dos egressos ${ }^{4,28}$.

Ter aptidão e afinidade com a especialidade também foi um fator relevante, como identificado em estudos nacionais e internacionais sobre a escolha da especialidade médica em geral. Nesse sentido, é fundamental a exposição dos graduandos à especialidade de MFC de forma qualificada e em vários momentos do curso para que essa afinidade possa ser despertada ${ }^{9,29}$. Entretanto, algumas dificuldades são relatadas para a inserção dos estudantes de Medicina nos cenários de prática em atenção primária de forma longitudinal, seja de cunho administrativo, logístico ou pela falta de profissionais da Estratégia Saúde da Família (ESF) que exerçam função de preceptoria.

A influência dos pais ou de outros membros da família na escolha da MFC, ao contrário de outras especialidades, não foi relevante. Esse fato

\begin{tabular}{|c|c|c|}
\hline \multicolumn{3}{|c|}{$\begin{array}{l}\text { Opinião de médicos que ingressaram na residência de Medicina } \\
\text { de Família e Comunidade no estado de Pernambuco, no período } \\
\text { de } 2012 \text { a } 2017 \text {, sobre os fatores que influenciaram na escolha da } \\
\text { especialidade - Recife, } 2018\end{array}$} \\
\hline $\begin{array}{c}\text { Fatores que influenciaram na escolha da } \\
\text { especialidade de MFC }\end{array}$ & $\begin{array}{l}\text { Ranking } \\
\text { médio }\end{array}$ & $\begin{array}{l}\text { Desvio } \\
\text { padrão }\end{array}$ \\
\hline \multicolumn{3}{|l|}{ ASPECTOS PESSOAIS } \\
\hline $\begin{array}{l}\text { Interesse em ajudar as pessoas (compromisso } \\
\text { social) }\end{array}$ & 4,5 & 0,61 \\
\hline Aptidão e afinidade com a especialidade & 4,3 & 0,77 \\
\hline $\begin{array}{l}\text { Momento da vida (circunstâncias familiares } \\
\text { ou pessoais) }\end{array}$ & 3,9 & 1,12 \\
\hline Influência dos pais ou de outros parentes & 1,9 & 1,05 \\
\hline Média do bloco & 3,2 & \\
\hline \multicolumn{3}{|l|}{ CARACTERÍSTICAS DA RESIDÊNCIA DE MFC } \\
\hline Duração da residência & 3,1 & 0,92 \\
\hline Disponibilidade de vagas de residência & 3,3 & 1,06 \\
\hline Média do bloco & 3,2 & \\
\hline \multicolumn{3}{|c|}{ CARACTERÍSTICAS DA ESPECIALIDADE DE MFC } \\
\hline Conteúdo técnico da especialidade & 4,1 & 0,76 \\
\hline Prestígio da especialidade & 1,7 & 0,85 \\
\hline Remuneração/retorno financeiro & 2,6 & 1,16 \\
\hline $\begin{array}{l}\text { Perspectiva de segurança profissional } \\
\text { (mercado de trabalho) }\end{array}$ & 2,9 & 1,25 \\
\hline Perspectivas de crescimento profissional & 3,3 & 1,13 \\
\hline Oportunidade de ensino e pesquisa & 3,4 & 1,11 \\
\hline Diversidade de casos/tipos de paciente & 4,2 & 0,95 \\
\hline $\begin{array}{l}\text { Possibilidade de trabalhar em horários } \\
\text { flexíveis }\end{array}$ & 2,9 & 1,35 \\
\hline Atmosfera/ambiente de trabalho & 3,8 & 1,11 \\
\hline $\begin{array}{l}\text { Oportunidade de realizar procedimentos } \\
\text { ambulatoriais }\end{array}$ & 3,6 & 1,05 \\
\hline $\begin{array}{l}\text { Carga horária média semanal do médico de } \\
\text { família }\end{array}$ & 3,6 & 0,99 \\
\hline Tipo de relação médico-paciente & 4,5 & 0,77 \\
\hline Trabalho em equipe multidisciplinar & 4,3 & 0,87 \\
\hline Estilo de vida que a especialidade propicia & 4,3 & 0,89 \\
\hline Assistência predominantemente ambulatorial & 4,1 & 0,86 \\
\hline Cuidado integral do paciente & 4,5 & 0,72 \\
\hline $\begin{array}{l}\text { Seguimento de pacientes em longo prazo } \\
\text { (longitudinalidade) }\end{array}$ & 4,5 & 0,69 \\
\hline $\begin{array}{l}\text { Preferência por cuidado primário versus } \\
\text { cuidado hospitalar }\end{array}$ & 4,3 & 0,93 \\
\hline Trabalho na comunidade & 4,1 & 1,04 \\
\hline Autonomia que a especialidade propicia & 3,3 & 1,17 \\
\hline Média do bloco & 3,4 & \\
\hline
\end{tabular}

$$
\begin{array}{l|l}
\hline \text { REVISTA BRASILEIRA DE EDUCAÇÃO MÉDICA } \\
\hline 44 \text { (3) : e078; } 2020
\end{array}
$$




\begin{tabular}{|c|c|c|}
\hline \multicolumn{3}{|l|}{$\begin{array}{c}\text { Tabela } 4 \\
\text { Continuação }\end{array}$} \\
\hline $\begin{array}{l}\text { Fatores que influenciaram na escolha da } \\
\text { especialidade de MFC }\end{array}$ & $\begin{array}{l}\text { Ranking } \\
\text { médio }\end{array}$ & $\begin{array}{l}\text { Desvio } \\
\text { padrão }\end{array}$ \\
\hline \multicolumn{3}{|c|}{ FATORES RELACIONADOS AO CURRÍCULO DA GRADUAÇÃO } \\
\hline $\begin{array}{l}\text { Influência de algum docente ou preceptor } \\
\text { que você admire }\end{array}$ & 3,1 & 1,32 \\
\hline Atividades práticas no âmbito da MFC & 3,1 & 1,39 \\
\hline $\begin{array}{l}\text { Módulos ou disciplinas teóricos relacionados } \\
\text { com MFC }\end{array}$ & 2,5 & 1,37 \\
\hline $\begin{array}{l}\text { Atividades extracurriculares relacionadas } \\
\text { com a especialidade }\end{array}$ & 2,8 & 1,37 \\
\hline $\begin{array}{l}\text { Participação em projetos de extensão e/ou } \\
\text { ligas acadêmicas }\end{array}$ & 2,1 & 1,09 \\
\hline $\begin{array}{l}\text { Participação em movimento estudantil ou } \\
\text { diretório acadêmico }\end{array}$ & 2,5 & 1,47 \\
\hline Participação em pesquisa/iniciação científica & 2,3 & 1,16 \\
\hline Média do bloco & 2,6 & \\
\hline
\end{tabular}

Fonte: Elaborada pelas autoras

pode estar relacionado com o ainda baixo prestígio social da especialidade relatado em alguns estudos, como também ao fato de ser uma residência que tem tido crescimento recente, portanto envolvendo uma geração mais jovem de médicos no Brasil ${ }^{19,20}$.

Em relação às características próprias da especialidade, todas as que estão em consonância com os princípios da atenção primária, como o cuidado integral do paciente, a pluralidade de casos, a assistência ambulatorial, o trabalho em equipe multidisciplinar, a longitudinalidade do cuidado e a atuação na comunidade, foram relevantes na escolha da carreira de $\mathrm{MFC}^{20}$.

Em contrapartida, o prestígio social da MFC, o retorno financeiro e o mercado de trabalho não foram sinalizados como fatores que tiveram impacto positivo na escolha da MFC, como observado em outros estudos ${ }^{12,29}$. Ressalta-se que alguns desses aspectos poderiam ser modificados a partir da instituição de políticas públicas que visassem à valorização da atenção primária e de planos de cargos, carreiras e salários para médicos de família no âmbito do SUS, sobretudo na $\mathrm{ESF}^{30}$.

No âmbito do currículo da graduação médica, a presença de preceptores modelos (role models) que exerceram papel inspirador no exercício da MFC relatada em vários países também esteve presente como uma influência neste estudo. Esse fato que está intrinsecamente ligado à vivência de atividades práticas no âmbito da MFC durante a graduação médica, aspecto igualmente destacado como influenciador pelos participantes ${ }^{23,25}$. Em contrapartida, nenhuma outra atividade acadêmica, curricular ou extracurricular, como aulas teóricas, ligas acadêmicas, projetos de extensão ou iniciação científica, foi apontada como influência positiva para a MFC. A análise dos aspectos relacionados ao currículo da graduação fica limitada no presente estudo, uma vez que isso não foi amplamente explorado. Entretanto, os achados reforçam a importância da inserção contínua em cenários de atenção primária e de MFC, além do papel do preceptor no processo de ensino e aprendizagem, assim como na escolha da especialidade.
Quando se analisaram os quatro blocos de fatores influenciadores da escolha da especialidade - aspectos pessoais, características da residência, características da especialidade e fatores relacionados ao currículo -, percebeu-se que, avaliados em conjunto, os fatores relacionados ao currículo da graduação não foram referidos pelos participantes como tendo influenciado na escolha da especialidade. Apesar de as DCN apontarem para uma formação com foco no cidadão, na família e na comunidade, com visão integral e contínua do cuidado, observa-se que a adequação dos cursos de Medicina a elas acontece de maneira heterogênea, seja por fatores ligados à própria IES, seja por fatores relacionados à rede de saúde ou ambos ${ }^{16,17}$.

\section{Características sociodemográficas}

A pesquisa Demografia médica no Brasil 2018 revelou que os homens ainda são maioria entre os médicos em atividade no país $(54,4 \%)$, entretanto existe uma clara tendência de feminização da profissão, pois as mulheres já são maioria entre os médicos mais jovens, representando $57,4 \%$ no grupo de até 29 anos e 53,7\% na faixa entre 30 e 34 anos. Observa-se essa mudança mais rápida em algumas especialidades, em que as mulheres já são maioria, como na MFC $(57,1 \%)$, fato que se verifica entre os participantes deste estudo e em outros países ${ }^{16,22}$. Essa feminização no âmbito da MFC é compreensível pelo fato de que mulheres médicas se adaptam melhor à atuação na atenção primária e ao trabalho em equipe multiprofissional, abordam mais aspectos preventivos, tendem a fazer menos uso desnecessário de tecnologias duras, estão mais propensas a uma boa relação médico-paciente e promovem maior envolvimento dos pacientes nas decisões relacionadas aos cuidados médicos ${ }^{16}$.

A média de idade dos especialistas em MFC é a menor de todas as especialidades no Brasil, o que indica a expansão recente da especialidade, que pode estar relacionada com a maior oferta de vagas de residência médica e/ou maior procura pela especialização. Solteiros e sem filhos predominaram na amostra, dados que estão em consonância com a baixa média de idade da especialidade no Brasil ${ }^{16}$.

Ser procedente de área rural ou de cidades de menor porte foi identificado entre as características de médicos que escolheram medicina de família em outros países, o que parece estar em acordo com os participantes do estudo, cuja maioria nasceu em cidades do interior do Brasil ${ }^{22,23}$.

\section{Perfil da instituição de graduação}

Em relação à distribuição da faculdade de origem, não houve diferença entre os egressos das três faculdades de Medicina da região metropolitana do estado de Pernambuco, considerando que o quantitativo anual de vagas no período analisado foi semelhante entre elas. Entretanto, a única faculdade localizada no interior do estado teve um quantitativo absoluto e percentual significativamente maior de egressos entre os participantes do estudo, apesar de disponibilizar menos vagas anuais no curso de Medicina quando comparada com as demais citadas ${ }^{31}$. Especulamos que a presença de faculdades de Medicina em cidades do interior, longe dos grandes centros urbanos, onde se concentram os complexos hospitalares superespecializados, seja um fator que influencie no aumento pela procura de uma especialidade básica essencialmente voltada para a atenção primária ambulatorial como a MFC. Outro aspecto importante a ser destacado é que essa universidade oferece vagas de residência de MFC, favorecendo a permanência dos egressos da faculdade na própria região em que se graduaram. 


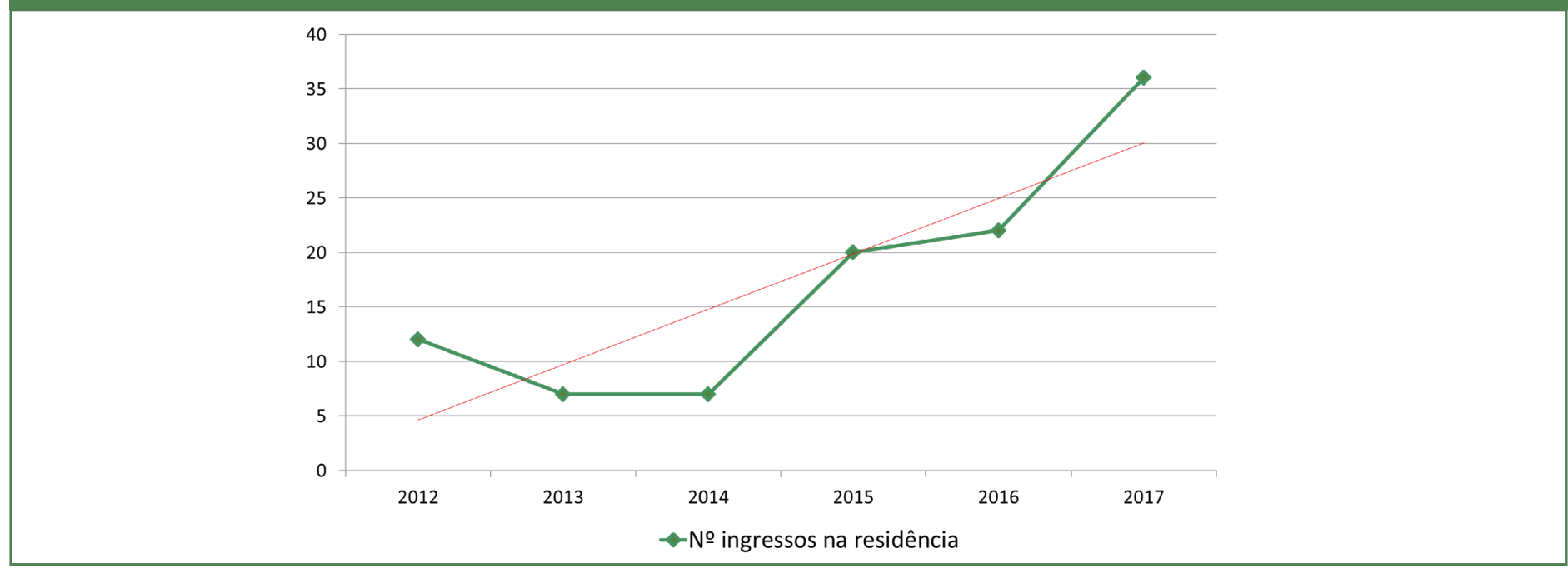

Fonte: Elaborada pelas autoras

Dos participantes $75 \%$ ingressaram na residência nos três anos finais do período do estudo e $25 \%$ nos três anos iniciais, o que pode sugerir uma tendência de crescimento na procura pela especialidade de MFC no estado, necessitando, entretanto, de estudos com outro desenho para confirmação (Gráfico 1).

Uma limitação do estudo refere-se à não análise dos currículos das faculdades do estado de Pernambuco, o que permitiria aprofundar a discussão sobre as diferentes influências que possam ter ocorrido de acordo com a inserção da MFC e APS ao longo dos cursos médicos.

\section{CONCLUSÃO}

Entre os fatores referidos pelos residentes como influenciadores na escolha da especialidade de MFC, incluem-se aspectos pessoais, características do programa de residência, assim como características da própria especialidade. Por sua vez, aspectos relacionados ao currículo da graduação de forma geral não contribuíram para a essa escolha, entretanto houve concordância de que a vivência em cenário de prática de MFC e o exemplo de preceptores modelos influenciaram na decisão pela especialidade. A qualificação de todos os ambientes de aprendizagem e o planejamento integrado do currículo com participação de profissionais de MFC podem contribuir para uma visão ampliada da atuação da especialidade de MFC desde a graduação.

Contudo, independentemente da especialidade que os graduandos terão na sua prática profissional futura, pensar em um currículo embebido da essência generalista, humana e integral permitirá aos egressos uma visão diferenciada do cuidado, com o compromisso social necessário para a transformação da prática médica vigente.

\section{REFERÊNCIAS}

1. Brasil. Ministério da Educação. Conselho Nacional de Educação. Câmara de Educação Superior. Resolução CNE/CES n ${ }^{\circ}$ 3, de 20 de junho de 2014. Institui diretrizes curriculares nacionais do curso de graduação em Medicina e dá outras providências. Diário Oficial da União, Brasília; 23 jun. 2014; Seção 1, p. 8.
2. Organización Panamericana de la Salud. La formación en medicina orientada hacia la atención primaria de salud. Washington, DC: Organización Panamericana de la Salud; c2018. Serie La renovación de la atención primaria de salud en las Américas [acesso em $16 \mathrm{dez}$ 2016]. Disponível em: http://new.paho.org/hq/dmdocuments/2010/ APS-Formacion_Medicina_Orientada_APS.pdf.

3. Gomes AP, Costa JRB, Junqueira T da S, Arcuri MB, Siqueira-Batista R. Atenção primária à saúde e formação médica: entre episteme e práxis. Rev. bras. educ. med. 2012;36(4):541-9.

4. Mullan F, Chen C, Petterson S, Kolsky G, Spagnola M. The social mission of medical education : ranking the schools. Acad Clin Ann Intern Med 2010;152(12):804-11.

5. Morley CP, Mader EM, Smilnak T, Bazemore A, Petterson S, Rodríguez JE, et al. The social mission in medical school mission statements: associations with graduate outcomes. Fam. med. 2015;47(6):427-34.

6. Costa JRB, Romano VF, Costa RR, Gomes AP, Alves LA, SiqueiraBatista R. A transformação curricular e a escolha da especialidade médica. Rev. bras. educ. med. 2014;38(1):47-58 [acesso em 2 maio 2020]. Disponível em: http://www.scielo.br/scielo.php?script=sci_ arttext\&pid=S0100-55022014000100007\&lng=pt\&nrm=iso\&tlng=en.

7. Ghuntla TP, Mehta HB, Gokhale PA, Shah CJ. Specialty preference and influencing factors for post graduate specialty selection of medical students: a study conducted in west zone of India. Al Ameen J Med Sci. 2015;8(2):202-5.

8. Lefevre JH, Roupret M, Kerneis S, Karila L. Career choices of medical students: a national survey of 1780 students. Med. educ. 2010;44:603-12.

9. Al-Ansari SS, Khafagy MA. Factors affecting the choice of health specialty by medical graduates. J Family Community Med. 2006;13(3):119-23.

10. Australian Medical Workforce Advisory Committee. Career decision making by doctors in vocational training. Sydney: AMWAC; 2003.

11. Australian Medical Workforce Advisory Committee. Career decision making by doctors in their postgraduate years - a literature review. Sydney: AMWAC; 2002.

$$
7 \mid \begin{array}{l|l}
\text { REVISTA BRASILEIRA DE EDUCAÇÃO MÉDICA } \\
\hline 4 \text { (3) : e078; } 2020
\end{array}
$$


12. Sociedade Brasileira de Medicina de Família e Comunidade. A Medicina de Família e Comunidade: o que, como, quando, onde, por quê? Rio de Janeiro: Sociedade Brasileira de Medicina de Família e Comunidade; 2004.

13. Starfield B, Shi L, Grover A, Macinko J. The effects of specialist supply on populations' health: assessing the evidence. Health aff. 2005;24 (Suppl 1):97-107.

14. Rohan-Minjares F, Alfero C, Kaufman A. How medical schools can encourage students' interest in Family Medicine. Acad. med. 2015;90(5):553-5.

15. Escorel S, Giovanella L, Mendonça MHM de, Senna M de CM. O Programa de Saúde da Família e a construção de um novo modelo para a atenção básica no Brasil. Rev. panam. salud pública 2007;21(2):16476 [acesso em 2 maio 2020]. Disponível em: http://www.scielosp.org/ $\mathrm{pdf} / \mathrm{rpsp} / \mathrm{v} 21 \mathrm{n} 2-3 / 11 . \mathrm{pdf}$.

16. Scheffer M. Demografia médica no Brasil 2018. São Paulo: FMUSP, CFM, Cremesp; 2018. v. 1, 286 p.

17. Augusto DK, David L, Oliveira DOPS, Trindade TG da, Lermen Junior N, Poli Neto P. Quantos médicos de família e comunidade temos no Brasil? Rev. bras. med. fam. comunidade 2018;13(40):1-4 [acesso em 2 maio 2020]. Disponível em: https://www.rbmfc.org.br/ rbmfc/article/view/1695.

18. Number of physicians by specialty and age, Canada, 2018. [acesso em 30 maio 2020]. Disponível em: https://www.cma.ca/sites/default/files/ pdf/Physician\%20Data/02-physicians-by-specialty-age-e.pdf

19. Rohan-Minjares F, Alfero C, Kaufman A. How medical schools can encourage students' interest in Family Medicine. Acad. med. 2015;90(5):553-5.

20. Harris MG, Gavel PH, Young JR. Factors influencing the choice of specialty of Australian medical graduates. MJA. 2005;183(6):295-300.

21. Mello GA, Mattos ATR, Souto BGA, Fontanella BJB, Demarzo MMP. Médico de família: ser ou não ser? Dilemas envolvidos na escolha desta carreira. Rev. bras. educ. med. 2009;33(3):464-71.

22. Gasiorowski J, Rudowicz E, Safranow K. Motivation towards medical career choice and future career plans of Polish medical students. Adv Heal Sci Educ 2015;20(3):709-25.

23. Erikson CE, Danish S, Jones KC, Sandberg SF, Carle AC. The role of medical school culture in primary care career choice. Acad. med. 2013;88(12):1919-26.

24. Bennett KL, Phillips JP. Finding, recruiting, and sustaining the future primary care physician workforce: a new theoretical model of specialty choice process. Acad. med. 2010;85(10 Suppl):81-8.

25. Rodríguez C, López-Roig S, Pawlikowska T, Schweyer F-X, Bélanger
E, Pastor-Mira MA, et al. The influence of academic discourses on medical students' identification with the discipline of Family Medicine. Acad. med. 2015;90:660-70.

26. Demarzo MMP, Almeida RCC de, Marins JJN, Trindade TG da, Anderson MIP, Stein AT, et al. Diretrizes para o ensino na atenção primária à saúde na graduação em Medicina. Rev. bras. educ. med. 2012;36(1):143-8.

27. Silva ATC da, Medeiros Junior ME de, Fontão P de N, Saletti Filho HC, Vital Junior PF, Bourget MMM, et al. Medicina de família do primeiro ao sexto ano da graduação médica: considerações sobre uma proposta educacional de integração curricular escola-serviço. Rev. bras. educ. med. 2017;41(2):336-45.

28. Dharamsi S, Ho A, Spadafora SM, Woollard R. The physician as health advocate: translating the quest for social responsibility into medical education and practice. Acad. med. 2011;86(9):1108-13.

29. Cruz JAS da, Sandy NS, Vannucchi TR, Gouveia ÉM, Passerotti CC, Bruschini $\mathrm{H}$, et al. Fatores determinantes para a escolha da especialidade médica no Brasil. Rev. med. (São Paulo) 2010;89(1):32-42.

30. Sociedade Brasileira de Medicina de Família e Comunidade. Carta de Brasília. SBMFC; 2018. [acesso em 2 de maio 2020]. Disponível em: https://www.sbmfc.org.br/noticias/carta-de-brasilia-confira-documentoproduzido-durante-o-forum-nacional-de-atencao-primaria/

31. Brasil. Ministério da Educação. Cadastro Nacional de Cursos e Instituições de Educação Superior - Cadastro e-MEC [acesso em 20 abr 2018]. Disponível em: http://emec.mec.gov.br/.

\section{CONTRIBUIÇÃO DAS AUTORAS}

Lucia Helena Guimarães Rodrigues foi responsável pela execução do projeto de pesquisa, pesquisa bibliográfica, coleta de dados, compilação dos resultados e elaboração do texto, das tabelas e do gráfico. Taciana Barbosa Duque foi responsável pela orientação da pesquisa, análise dos resultados e supervisão da pesquisa. Reneide Muniz da Silva foi responsável pela orientação da pesquisa e correção do texto.

\section{CONFLITO DE INTERESSES}

As autoras declaram não haver conflito de interesses neste estudo.

\section{ENDEREÇO PARA CORRESPONDÊNCIA}

Lucia Helena Guimarães Rodrigues. Rua Martins Ribeiro, 381, Hipódromo, Recife, PE, Brasil. CEP: 52041-720.

E-mail: lucia.rodrigues@imip.org.br 